Research Article

\title{
Exosomes Secreted by Umbilical Cord Blood-Derived Mesenchymal Stem Cell Attenuate Diabetes in Mice
}

\author{
Rajni Sharma, ${ }^{1}$ Manju Kumari, ${ }^{1}$ Suman Mishra, ${ }^{1}$ Dharmendra K. Chaudhary, ${ }^{1}$ \\ Alok Kumar, ${ }^{1}$ Batia Avni, ${ }^{2,3}$ and Swasti Tiwari ${ }^{1}{ }^{1}$ \\ ${ }^{1}$ Department of Molecular Medicine \& Biotechnology, Sanjay Gandhi Post Graduate Institute of Medical Sciences, \\ Lucknow 226014, India \\ ${ }^{2}$ Department of Bone Marrow Transplantation and Cancer Immunotherapy, Hadassah-Hebrew University Medical Center, \\ Ein Kerem, Jerusalem, Israel \\ ${ }^{3}$ Faculty of Medicine, Hebrew University of Jerusalem, Israel
}

Correspondence should be addressed to Swasti Tiwari; tiwaris@sgpgi.ac.in

Received 5 August 2021; Revised 21 October 2021; Accepted 22 November 2021; Published 10 December 2021

Academic Editor: Sanjay K. Banerjee

Copyright (c) 2021 Rajni Sharma et al. This is an open access article distributed under the Creative Commons Attribution License, which permits unrestricted use, distribution, and reproduction in any medium, provided the original work is properly cited.

\begin{abstract}
Mesenchymal stem cell (MSC) therapy is an innovative approach in diabetes due to its capacity to modulate tissue microenvironment and regeneration of glucose-responsive insulin-producing cells. In this study, we investigated the role of MSC-derived exosomes in pancreatic regeneration and insulin secretion in mice with streptozotocin-induced diabetes. Mesenchymal stem cells (MSCs) were isolated and characterized from umbilical cord blood (UCB). Exosomes were isolated and characterized from these MSCs. Diabetes was induced in male C57Bl/6 mice by streptozotocin (STZ; $40 \mathrm{mg} / \mathrm{kg}$ body weight, i.p.) for five consecutive days. The diabetic mice were administered (i.v.) with MSC $\left(1 \times 10^{5}\right.$ umbilical cord blood MSC cells/mice/day), their derived exosomes (the MSC-Exo group that received exosomes derived from $1 \times 10^{5} \mathrm{MSC}$ cells/mice/ day), or the same volume of PBS. Before administration, the potency of MSCs and their exosomes was evaluated in vitro by T cell activation experiments. After day 7 of the treatments, blood samples and pancreatic tissues were collected. Histochemistry was performed to check cellular architecture and $\beta$ cell regeneration. In body weight, blood glucose level, and insulin level, cell proliferation assay was done to confirm regeneration of cells after MSC and MSC-Exo treatments. Hyperglycemia was also attenuated in these mice with a concomitant increase in insulin production and an improved histological structure compared to mice in the PBS-treated group. We found increased expression of genes associated with tissue regeneration pathways, including Reg2, Reg3, and Amy $2 b$ in the pancreatic tissue of mice treated with MSC or MSC-Exo relative to PBS-treated mice. MicroRNA profiling of MSC-derived exosomes showed the presence of miRs that may facilitate pancreatic regeneration by regulating the Extl3-Reg-cyclinD1 pathway. These results demonstrate a potential therapeutic role of umbilical cord blood MSC-derived exosomes in attenuating insulin deficiency by activating pancreatic islets' regenerative abilities.
\end{abstract}

\section{Introduction}

Diabetes is a major growing health problem that has been estimated to afflict well over 300 million people globally. The two most common types of diabetes are type 1 (T1DM) and type 2 diabetes mellitus (T2DM). Together, they account for a large and growing patient population with pancreatic $\beta$ cell deficiency. In the case of T1DM, $\beta$ cell deficiency is associated with autoimmune destruction of $\beta$ cells, while in the case of T2DM, it is associated with $\beta$ cell dys- function $[1,2]$. Nevertheless, substantial $\beta$ cell loss results in permanent endocrine deficiency and irreversible diabetes due to their limited regenerative capacity in adults [3]. Thus, any treatment modality to restore $\beta$ cell mass would be beneficial for all forms of diabetes $[4,5]$. Mesenchymal stem cells (MSCs) present a promising approach for restoring $\beta$ cell mass in diabetes $[6,7]$. Immune modulatory properties of MSCs have been attributed to their therapeutic effects in T1DM $[8,9]$. Bone marrow-derived MSCs have been found to inhibit $\mathrm{T}$ cell proinflammatory response to islet antigen 
GAD65, in patients with T1DM $[10,11]$. Moreover, these MSCs were shown to affect the antigen-presenting function of dendritic cells (DC) by promoting an immature DC phenotype [12]. The immunomodulatory effects of autologous bone marrow MSCs in patients with recent onset T1DM showed preservation of $\mathrm{C}$ peptide levels in response to a mixed meal tolerance test (MMTT) [13]. Similar immunomodulatory potential of MSCs derived from other sources, such as umbilical cord blood (UCB), will have a profound impact in developing therapeutic modalities for diabetes due to their availability and noninvasive nature of collection [14-16]. The mechanisms of action of MSCs have been attributed to their secretome rather than to their transdifferentiation [17]. However, MSC administration for therapeutic purposes may expose individuals to the side effects of cell-based therapy and immune rejection [18]. This has geared a great scientific interest in exploring alternative approaches inspired by the paracrine hypothesis in regenerative medicine, namely, using MSC-derived extracellular vesicles, such as exosomes, rather than the cells themselves. Exosomes have been shown to play a key role in cell-tocell communication. Ample of data suggests that exosomes may transfer beneficial outputs from cells to neighboring diseased or injured cells through the delivery of biomolecules with therapeutic potential. The protective effect of UCB-derived MSC-secreted exosomes has been studied in myocardial repair and autoimmune diseases $[14,16]$. However, the potential of UCB-MSCs and their derived exosomes in attenuating endocrine deficiencies caused due to nonautoimmune pancreatic $\beta$ cell loss needs to be tested. In this study, we aimed to understand the therapeutic effect of exosomes secreted by UCB-derived MSC on pancreatic repair/regeneration in diabetic mice, where streptozotocininduced pancreatic beta cell destruction leads to diabetes.

\section{Materials and Methods}

2.1. Isolation and Characterization of Human UCB-Derived MSCs. MSCs were isolated from human UCB as previously described [17]. The protocol has been approved by Institutional Committee for Stem Cell Research (IC-SCR; Code: 2019-03-IMP-08). MSCs propagated to the $3^{\text {rd }}-8^{\text {th }}$ passages were characterized using accepted MSC-positive markers anti-CD73, CD90, and CD105 (BioLegend, San Diego, CA, USA), negative hematopoietic markers anti-CD45 and CD34 (BioLegend, San Diego, CA, USA), HLA-DR (BD Biosciences, San Jose, CA, USA) by confocal microscopy (FluoView F10i confocal microscope, Olympus), and flow cytometry (Becton Dickinson, New Jersey, USA). Negative gates were set relative to isotype controls. No chromosomal aberrations were observed at high passage (data not shown). In vitro differentiation was performed using StemPro ${ }^{\mathrm{TM}}$ Adipogenesis Differentiation Kit (A1007001, Thermo Scientific, Pittsburg, PA Scientific, USA), StemPro ${ }^{\mathrm{TM}}$ Osteogenesis Differentiation Kit (A1033201, Thermo Scientific, Pittsburg, PA Scientific, USA), and StemPro ${ }^{\mathrm{TM}}$ Chondrogenesis Differentiation Kit (A1007101, Thermo Scientific, Pittsburg, PA Scientific, USA) [19, 20].
2.2. Isolation and Characterization of Exosomes from Human UCB-Derived MSCs (MSC-Exo). Exosomes were enriched by differential centrifugation as described previously [21]. Briefly, samples were centrifuged at $17,000 \mathrm{~g}$ for $10 \mathrm{~min}$ at $4^{\circ} \mathrm{C}$ followed by ultracentrifugation (Beckman Coulter $\mathrm{LE} 80 \mathrm{~K}, \mathrm{CA}, 362$, USA) at $126,000 \mathrm{~g}$ for $2 \mathrm{~h}$ at $4^{\circ} \mathrm{C}$. The exosomal pellet was resuspended in an isolation buffer (PBS). Isolated exosomes were characterized for size using dynamic light scattering (DLS) analysis. The sample temperature was allowed to equilibrate for $10 \mathrm{~min}$ before each measurement and was shaken for $20 \mathrm{~min}$ at $37^{\circ} \mathrm{C}$ to dissolve any aggregation, followed by DLS measurements at $20^{\circ} \mathrm{C}$ using a Zetasizer Nano (Malvern Instruments Ltd., UK). The light scattering was recorded for $200 \mathrm{~s}$ with 10 replicate measurements. DLS signal intensity was calculated by the Dispersion Technology Software v.5.10 (Malvern Instruments Ltd., UK). The mean hydrodynamic diameter of exosomes was calculated by fitting a Gaussian function. The peak maximum of the Gaussian function was used to estimate exosome size. Gaussian fitting, mean value, and standard deviation were calculated and compared using Origin Pro 9.0.0 (Origin Lab Corp, USA). Shape characterization was done by scanning electron microscopy (SEM). MSC-Exo suspensions were mixed $1: 1$ with $4 \%$ paraformaldehyde and were then applied to 200-mesh nickel grids. They were dried and coated with gold particles, and images were taken by scanning electron microscope (JSM 6490).

$\mathrm{T}$ cell activation analysis was performed by using mouse spleen (source of $\mathrm{T}$ cell) and followed by $\mathrm{CD}^{+} \mathrm{T}$ cell enrichment column (cat MTCC-25, R\&D systems, USA) as per the manufacturer's instruction. Proliferation analysis was performed on (i) unactivated $\mathrm{T}$ cells, (ii) activated $\mathrm{T}$ cells, (iii) activated T cells treated with MSCs, and (iv) activated $\mathrm{T}$ cells treated with MSC-Exo. For this, $1 \times 10^{5} \mathrm{~T}$ cells were seeded in 10\% FBS (exosome free) RPMI media in 96well plates. Cells were activated by using $50 \mu \mathrm{l}$ of $5 \mu \mathrm{g} / \mathrm{ml}$ anti-CD3 antibodies (or 2C11) (BioLegend, San Diego, CA, USA) for 4 days at $37^{\circ} \mathrm{C}$. Similarly, in the treated group, these activated $\mathrm{T}$ cells were cocultured with MSCs $\left(5 \times 10^{4}\right.$ cells/well) and MSC-derived exosome (collected from $5 \times$ $10^{4}$ MSCs cells for $24 \mathrm{~h}$ ). After 4 days, nonadherent T cells were collected and live $\mathrm{T}$ cell counting was performed by using trypan blue and haemocytometer.

2.3. Microarray Analysis for MicroRNA Profile. For microarray analysis, RNA was isolated from MSC and MSC-Exo by miRNAeasy kit (Qiagen India Pvt. Ltd.) as per the manufacturer's protocol. The quantity was checked by NanoDrop ND-2000 (Thermo Fisher Scientific, Pittsburgh, PA). Samples with A260/280 more than 1.8 were subjected to microarray analysis by GeneChip ${ }^{\mathrm{TM}}$ miRNA 4.0 Array according to the manufacturer's instruction (Thermo Fisher Scientific). Transcriptome Analysis Console program (version 4.0.0.25, Applied Biosystems) conducted a statistical analysis of the output files (.CEL). Conditions used were background correction, quantile, normalization, description, and $\log _{2}$ value conversion using the RMA+DABG algorithm. Data represented as heat map and volcano plot. 
2.4. In Vivo Diabetic Mouse Model Development. 8-week-old male C57Bl/6 mice (body weight $20 \mathrm{~g}$ ) were used throughout the study. The protocol has been approved by Institutional Animal Ethics Committee (Ref. no: P-03/20/2017). Mice were procured from CSIR-Indian Institute of Toxicology Research, Lucknow, and were housed at the animal care facility of Sanjay Gandhi Postgraduate Institute of Medical Science, Lucknow. All mice were housed under the specific pathogen-free conditions in individually ventilated cages with $12 \mathrm{~h}$ light and dark cycle. All mice received drinking water and diet ad libitum. To induce T1DM, mice were injected intraperitoneal with multiple-dose injections of streptozotocin (STZ) $40 \mathrm{mg} / \mathrm{kg}$ body weight, freshly dissolved in $0.1 \mathrm{mmol} / \mathrm{l}$ sodium citrate ( $\mathrm{pH} 4.5$ ) for 5 consecutive days as previously described [22]. Blood samples for glucose levels monitoring were collected daily (from day 1 to 10) from tail vein using a glucometer (Elite Diabetes Care System). Animals were considered diabetic when their blood glucose levels were $\geq 200 \mathrm{mg} / \mathrm{dl}$. The control nondiabetic mouse group received a corresponding volume of $0.1 \mathrm{mmol} / \mathrm{l}$ of sodium citrate ( $\mathrm{pH} 4.5$ ) (Figure 1). Insulin was measured on pancreatic tissue using mouse insulin ELISA kit (Thermo Scientific, Pittsburg, PA Scientific, USA) on day 10 and day 17 in the control and diabetic mice treated with PBS, MSCs, or MSC-Exo ( $n=6 /$ group).

2.5. Proliferation Assays in Diabetic Mice Administered with MSCs and MSC-Exo. After 10 days of STZ treatment, mice were randomly divided into three groups: (i) vehicle group that received i.v. PBS injections from day 11 to day 17 $(n=6)$, (ii) MSC group that received i.v. injection of $1 \times$ $10^{5}$ MSC cells/mice from day 11 to day $17(n=6)$, (iii) Exo group that received i.v. injection of MSC-Exo derived from $1 \times 10^{5} \mathrm{MSCs} / \mathrm{mice}$ from day 11 to day $17(n=6)$. To investigate the homeostatic turnover of pancreatic cells in all three groups, two doses of $\mathrm{BrdU}$ (at $2 \mathrm{~h}$ and $16 \mathrm{~h}$ ) were administered at day 18 (three mice for each group, BrdU at $50 \mu \mathrm{g} / \mathrm{kg}$ in saline, i.p.) (Sigma-Aldrich, St. Louis, Missouri, USA).

2.6. Histology. Mice were euthanized on day 18 by cervical dislocation. For excision of the pancreas, mice were perfused with 1x PBS. Pancreases were carefully cleaned to remove excess fat tissue, fixed in $4 \%$ buffered paraformaldehyde (PFA) and then embedded in paraffin wax for histological processing. Paraffin-embedded tissues were sectioned $(5 \mu \mathrm{m}$ thickness) using a microtome (Thermo Scientific, Pittsburg, PA Scientific, USA). The sections were then processed for histological and/or immunohistochemical analysis.

For histopathogical analysis, five random fields were selected on three different sections from each group and the number of cells were counted as healthy and damaged on the basis of cellular morphology.

2.7. Proliferation Assay. Proliferating cells in pancreas sections were detected by indirect immunoperoxidase test as described previously [23]. Briefly, the sections ( $5 \mu \mathrm{m}$ thickness) were deparaffinized using xylene and rehydrated by descending gradings of ethanol. The sections were washed in 1x PBS and were further processed for heat-mediated antigen retrieval by using antigen-unmasking solution (Vector Labs, USA). The sections were washed with $1 \mathrm{x}$ PBS followed by blocking with $3 \%$ bovine serum albumin (BSA, Sigma-Aldrich, St. Louis, Missouri, USA). The sections were then probed with anti-BrdU antibody (B2531, SigmaAldrich, St. Louis, Missouri, USA) overnight at $4^{\circ} \mathrm{C}$. In the next morning, the sections were washed with $1 \mathrm{x}$ PBS and incubated with HRP-tagged anti-mouse secondary antibody for $1 \mathrm{~h}$ at $37^{\circ} \mathrm{C}$. The sections were then washed for three times with $1 \mathrm{x}$ PBS, and color was developed by $3,3^{\prime}$-diaminobenzidine $(\mathrm{DAB})$ chromogen solution $\left(106038, \mathrm{GeNei}^{\mathrm{TM}}\right)$ at $37^{\circ} \mathrm{C}$ for $10 \mathrm{~min}$. Sections were counterstained with haematoxylin, dehydrated in ethanol gradings, and mounted in DPX. Images were captured on an Olympus IX73 microscope and analysed by ImageJ software.

2.8. Western Blotting. Exosomes were lysed in RIPA buffer with a freshly added protease inhibitor cocktail (Roche Diagnostics, Germany). Protein concentrations were determined by BCA (Pierce, BCA Protein Assay Kit, Thermo Scientific, Pittsburg, PA). Equal amount of proteins was denatured and resolved on denaturing SDS-PAGE gel (12\%) and transferred to PVDF membrane. To prevent any protein loss due to stripping and for the ease of interpretation, membrane was cut into the lane after transfer of gel. The membrane was probed overnight with mouse anti-TSG101 (ab125011, Abcam, Cambridge, MA, USA), anti-CD63 (ab59479, Abcam), and anti-CD81 (ab109201, Abcam, Cambridge, MA, USA) antibody followed by HRP-conjugated secondary antibody. The membrane was visualized by an enhanced chemiluminescence detection system (Clarity ${ }^{\mathrm{TM}}$ western ECL substrate). Images were acquired on a ChemiDoc imaging system (Universal Hood III, Bio-Rad, California, USA).

2.9. Next-Generation Sequencing for Transcriptome Profile. RNA was extracted from diabetic mouse pancreas treated with PBS, MSCs, and MSC-Exo for sequencing and qPCR experiments. The RNA quality was tested using $1 \%$ agarose gel electrophoresis, quantities were measured by Qubit system (Invitrogen), and absorbance ratios (A260/280 and A260/230) were tested using NanoDrop ND-2000 (Thermo Fisher Scientific, Pittsburgh, PA, USA). Samples with A260/ 280 and A260/230 ratios more than 2.0 were used for sequencing using a MinION sequencer according to the manufacturer's instructions (Oxford Nanopore Technologies). In brief, the RNA samples (50 ng per sample) were reverse transcribed with a cDNA-PCR sequencing kit (SQK-PCS109); then, the samples were barcoded with a PCR barcoding kit (SQK-PBK004). Samples were primed with a flow cell priming kit (FLP001) and loaded on MinION flow cells, according to the manufacturer's instruction (Oxford Nanopore Technologies). MinKNOW was used to convert the generated Fast_5 files into FastQ format. Fold expressions were summarised in a heat map using HeatMapper software (http://www.heatmapper.ca/). Pathway analysis was performed using Reactome (https://reactome .org/), KEGG (http://www.genome.jp/kegg/), and Panther (http://pantherdb.org/). 


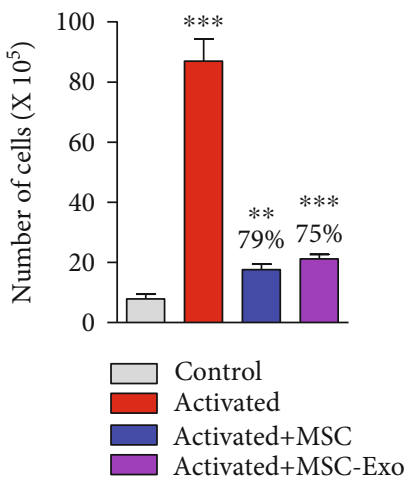

(a)

(i)

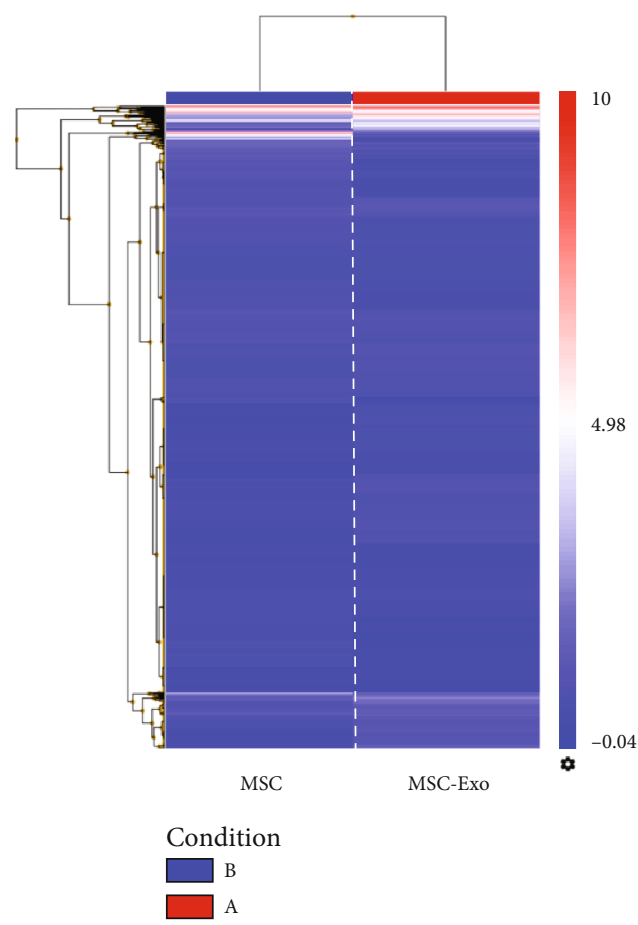

(ii)

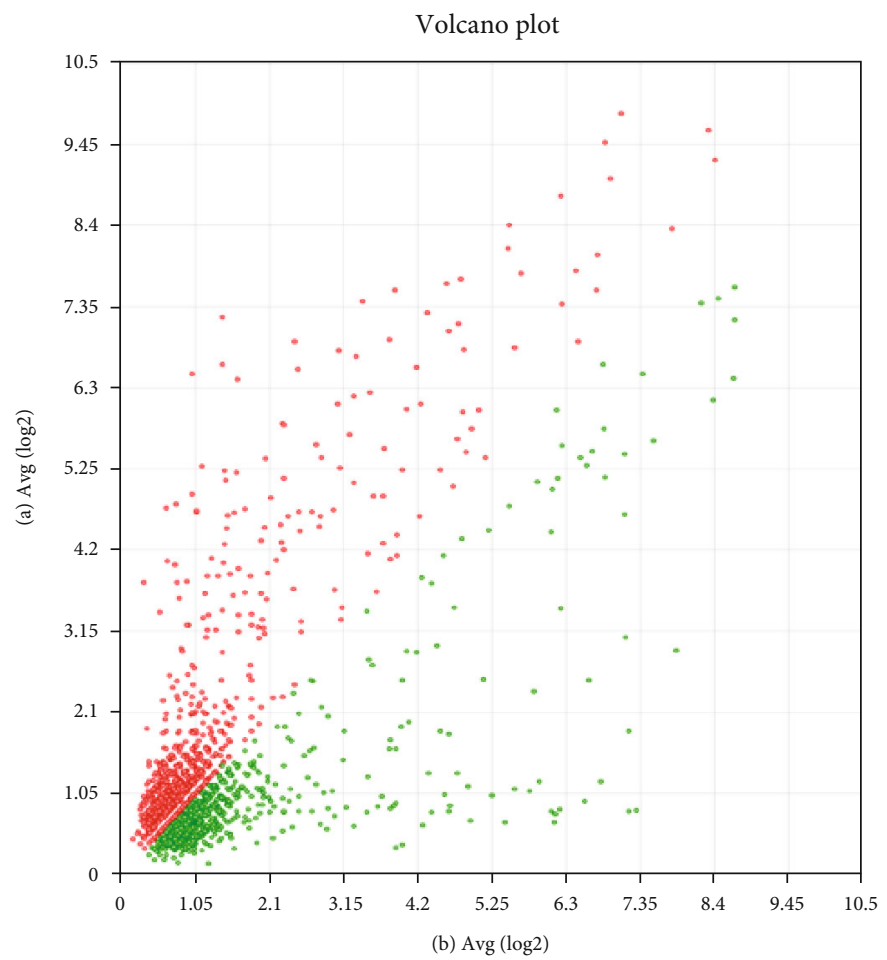

(b)

FIGURE 1: Human UCB-MSC and their derived exosomes showed similar microRNA profile and function. (a) Histogram representing T cell activation or proliferation in vitro attenuated after treatment with MSCs and MSC-Exo. Data are shown as the mean \pm SD. The significance of differences between mean values was estimated by Student's $t$-test (unpaired, two-tailed). ${ }^{*} p<0.05$ and ${ }^{* * *} p<0.001$ compared to control. (b) (i) Heat map and (ii) volcano plot representing the comparative miRNA profile of MSCs and MSC-Exo.

2.10. Real-Time PCR. cDNA synthesis was performed with $1 \mu \mathrm{g}$ of total RNA template per sample (the PBS, MSC, and MSC-Exo groups) by cDNA RT kit (Thermo Scientific, Pittsburgh, PA, USA). The protocol was used according to the manufacturer's instructions. Real-time PCR was performed using an ABI 7500 Sequence Detection System (Applied Biosystems, California, USA) in the presence of SYBR Green Master Mix (Takara Bio, Shiga, Japan). Standard PCR conditions were used as prescribed in the reagent protocol.

The primer sequences used were as follows: Reg3 forward primer-5'-GAATATACCCTCCGCACGCA- $3^{\prime}$ and
Reg3 reverse primer-5' -TCTTTTGGCAGGCCAGTTCT-3', Reg2 forward primer-5' ${ }^{\prime}$ AATCAACTGCCCAGAGGGTG$3^{\prime}$ and Reg2 reverse primer-5' -GCCACAAAGTTGCTCTCA GC-3', Amy $2 b$ forward primer- $5^{\prime}$-TGGGAGGACTGCTA TTGTCC- $3^{\prime}$ and $A m y 2 b$ reverse primer- $5^{\prime}$-CATTGTTGC ACCTTGTCACC-3', TLR4 forward primer-5'-ATGCAT GGATCAGAAACTCAGCAA- $3^{\prime}$ and TLR4 reverse primer$5^{\prime}$-AAACTTCCTGGGGAAAAACTCTGG-3' , and $18 \mathrm{~S}$ forward primer- $5^{\prime}$-GGCCCTGTAATTGGAATGAGTC- $3^{\prime}$ and $18 \mathrm{~S}$ reverse primer- $5^{\prime}$-CCAAGATCCAACTACGAGCTT-3' 

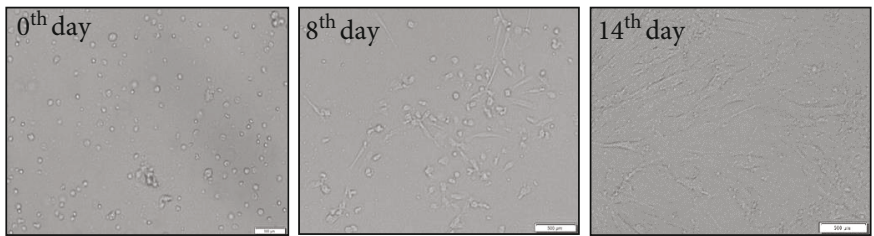

(a)
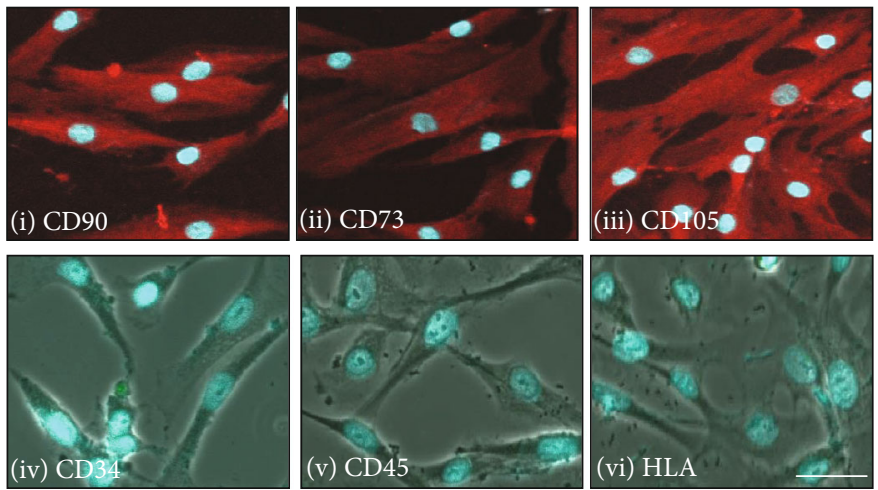

(b)
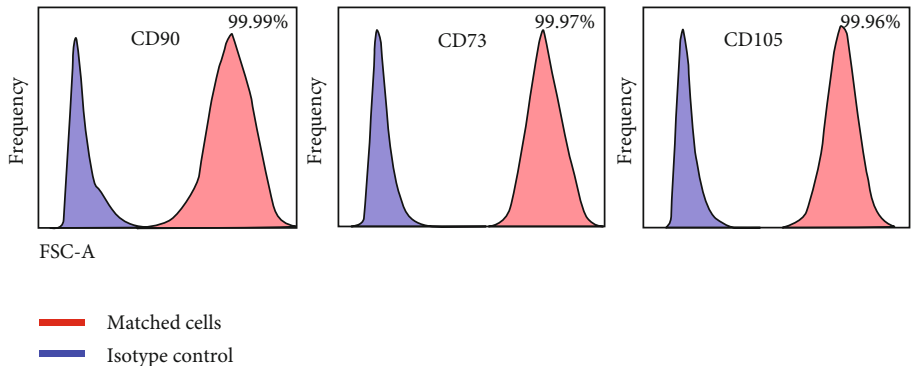

(c)

FIGURE 2: Characterization of human UCB-derived mesenchymal stem cells (MSCs). (a) Typical fibroblastic morphology of MSCs (i) at day of seeding, (ii) at day 8 , and (iii) at day 14 of seeding. Scale bar $=500 \mu \mathrm{M}$. (b) Confocal microscopy images showing expression of (i) CD90, (ii) CD73, and (iii) CD105 in isolated MSCs and lack of expression of (iv) CD34, (v) CD45, and (vi) HLA-DR in isolated MSCs. Scale bar $=100 \mu \mathrm{M}$. (c) Flow cytometry showed expression of typical MSC surface markers CD90, CD73, and CD105. FSC-A: forward scattering area.

. Gene expression was calculated relative to the endogenous control sample (18S). Fold change was calculated relative to the PBS control group using the $2^{-\Delta \Delta \mathrm{Ct}}$ method (where $\mathrm{Ct}$ is the threshold cycle).

2.11. Statistical Analysis. GraphPad Prism 5 software was used for the statistical analysis. Data are presented as the mean \pm standard deviation. The significance of differences between mean values was estimated using two-tailed unpaired Student's $t$-test. The statistical significance was set at ${ }^{*} p<0.05,{ }^{* *} p<0.01$, and ${ }^{* * *} p<0.001$ vs. their respective control.

\section{Results}

3.1. Immunophenotypic Characterization and Differentiation of Human UCB-Derived MSCs. MSCs isolated from human UCB (hUCB) were identified by their fibroblast spindleshaped morphology (Figure 2(a)). Expressions of typical MSC markers (Figure 2(b, i-iii)) and absence of staining for hematopoietic markers (Figure 2(b, iv-vi)) were observed using immunofluorescence. The results were confirmed by FACS analysis for the presence of MSC markers CD73 (96\%), CD90 (99\%), and CD105 (99.9\%) (Figure 2(c)). Differentiation assays performed on isolated MSCs exhibited as expected trilineage differentiation potential (Figures 3(a)-3(c)). Adipogenic differentiation was confirmed by the positive Oil Red O staining of neutral lipid vacuoles in the cells on day 14 of culture. Under osteogenic differentiation media, the cells exhibited nodular calcium deposition, which was stained with alizarin red staining on day 21 of culture. Four weeks under chondrogenic conditions, the cells exhibited typical chondrocyte-like lacunae. Alcian Blue staining confirmed the presence of secreted glycosaminoglycans.

3.2. Human UCB-MSCs (MSC) and Their Derived Exosomes (MSC-Exo) Showed Similar MicroRNA Profile and Function. Isolated exosomes were characterized by their cup-shaped spheroidal morphology (size: $\sim 132 \mathrm{~nm}$ ) (Figure 3(d, i and ii)) 


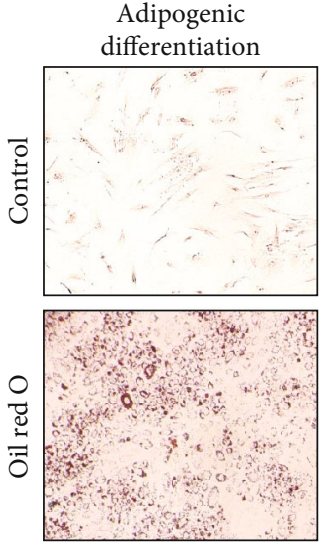

(a)

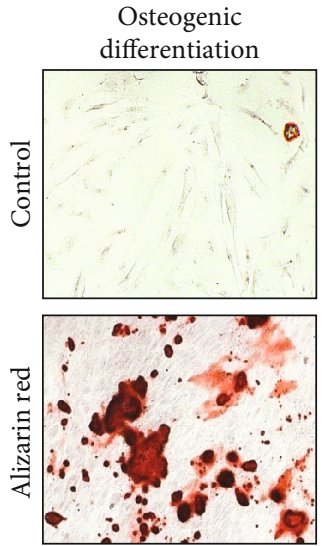

(b)

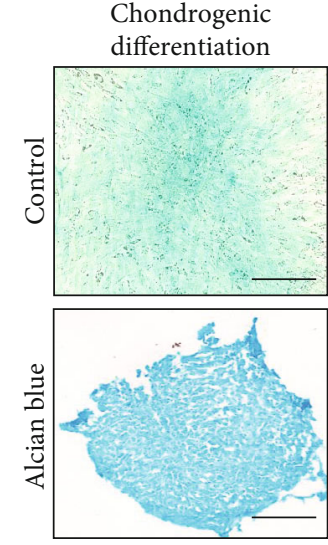

(c) (i) exosomes characterization"

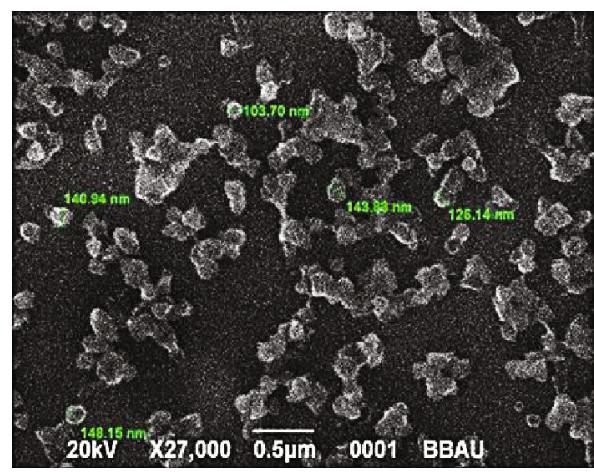

(ii)

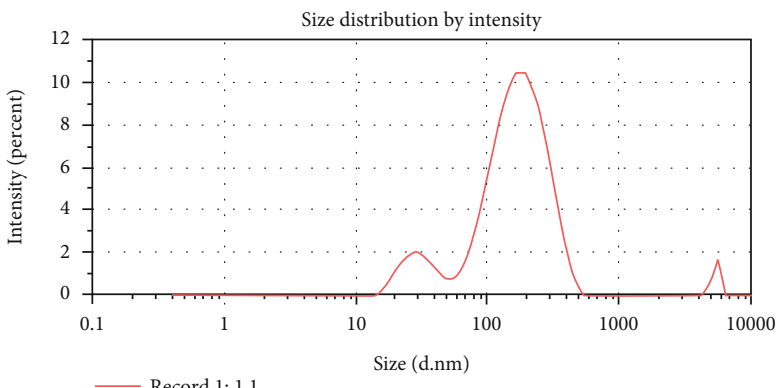

(d)

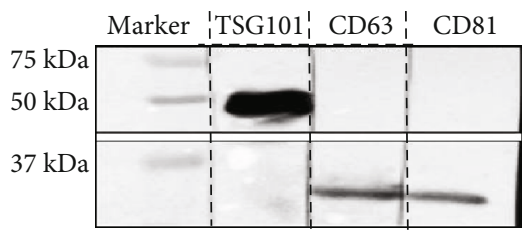

(e)

FIgURE 3: Characterization of MSCs and MSC-Exo. Representative photomicrographs of human umbilical cord blood-derived mesenchymal stem cells differentiated into (a) adipocytes, (b) osteocytes, and (c) chondrocytes and stained with Oil Red, Alizarin Red, and Alcian Blue stain, respectively. Scale bar $=100 \mu \mathrm{M}$. (d) (i) Electron micrograph of an MSC-Exo showing that $92.7 \%$ of the exosomes were between 50 and $140 \mathrm{~nm}$ (indicated by green letter). Scale bar $=0.5 \mu \mathrm{M}$. (ii) MSC-derived exosome size ranging between 100 and $140 \mathrm{nM}$ by dynamic light scattering analysis. (e) Representative immunoblot showing exosome-specific markers TSG101, CD63, and CD81 in isolated exosomes. Three independent experiments were performed. Full blot is submitted as a supplementary file.

and the presence of exosome-specific markers, i.e., TSG101, CD63, and CD81 proteins (Figure 3(e)). The heat map and volcano plots generated after microarray analysis showed a similar microRNA profile of MSC-Exo and MSCs (Figure 1(b, i and ii)). The expression patterns of miR-let 7a-5p, miR-24-3p, miR-2115-5p, miR-4156, miR-663a, miR-424-5p, miR-30d-5p, miR-450-p, miR-23b-5p, and miR19b-1-3p were similar between MSC-EXO and MSCs.

Suppressive effects of MSCs and their derived exosomes were tested on 2C11 antibody-activated T cells cocultured in the presence/absence of MSCs and MSC-Exo. 2C11 antibody was shown to induce $\mathrm{T}$ cell proliferation which was significantly attenuated by MSC-Exo $(p<0.05)$ and MSCs $(p<0.05)$ treatments, compared to control (Figure 1(a)).

3.3. In Vivo Mouse Model Induction of Diabetes and Pancreatic Injury by STZ Treatment. The schematic study design is shown in Figure 4. STZ-injected animals had significantly higher blood glucose as compared to vehicleinjected animals' hours of the initial STZ injection 


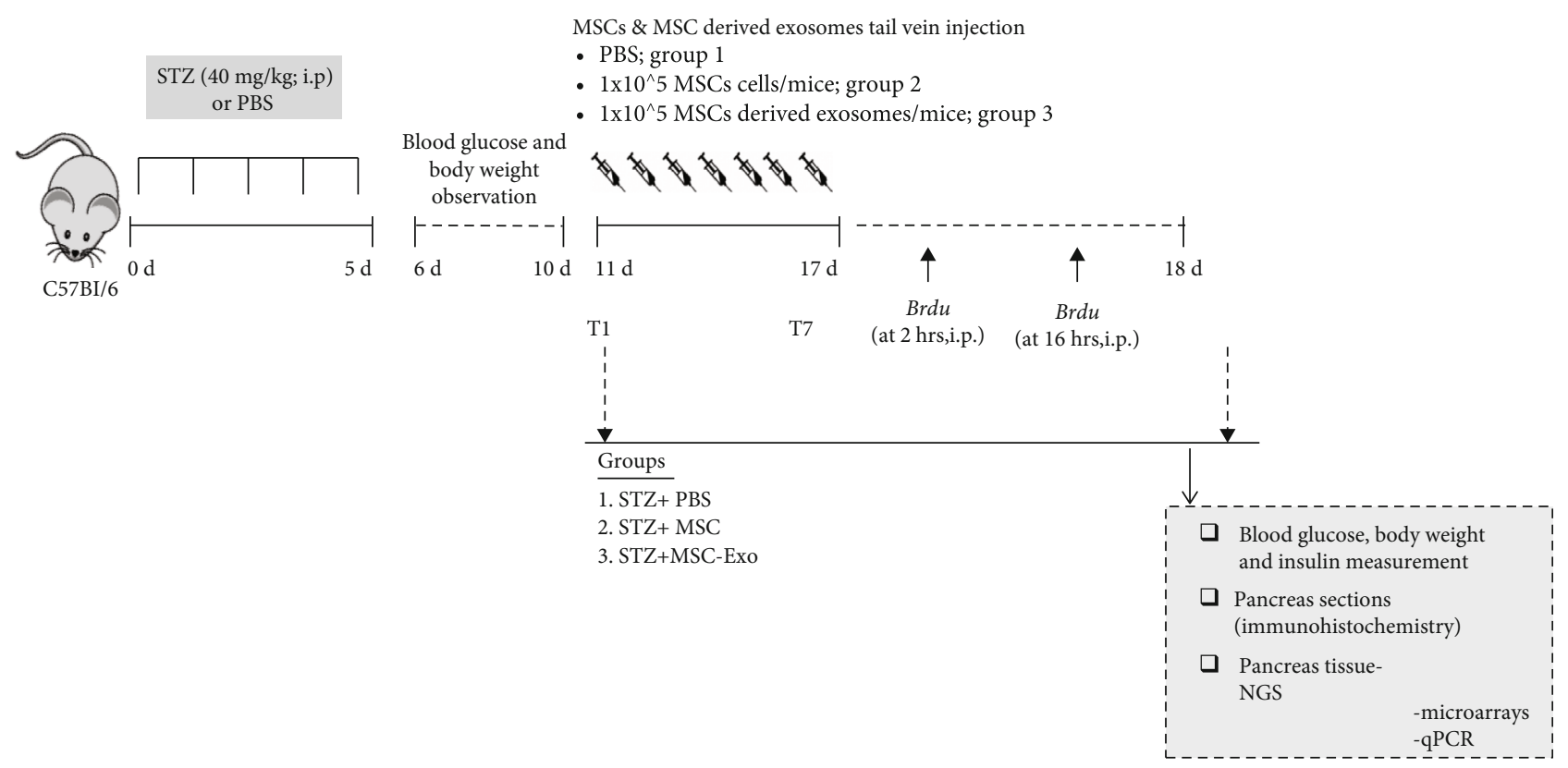

Figure 4: Timeline of the in vivo experimental design. Schematic illustration of the experimental plan. (a) In brief, 8-week-old adult male C57BL/6 mice (body weight $20 \mathrm{~g}$ ) were used throughout the study. To induce diabetes, mice were injected with multiple low-dose injections of streptozotocin intraperitoneal (i.p.) (STZ; $40 \mathrm{mg} / \mathrm{kg}$ freshly dissolved in $0.1 \mathrm{mmol} / \mathrm{l}$ sodium citrate (pH 4.5 )) for 5 consecutive days. Blood samples were collected from tail-vein and were used to monitor glucose levels. Animals were considered diabetic when their blood glucose levels were $\geq 200 \mathrm{mg} / \mathrm{dl}$. Mice in the nondiabetic control group received a corresponding volume of sodium citrate buffer alone. On day 10 , after STZ treatment, mice were randomly divided into three groups: (i) STZ+PBS group that received i.v. injection of PBS ( $n=6$ ), (ii) STZ +MSC group that received i.v. injection of $1 \times 10^{5}$ UCB-MSC cells/mice $(n=6)$, and (iii) STZ+MSC-Exo group that received i.v. injection of exosomes derived from $1 \times 10^{5} \mathrm{MSCs} /$ mice from day 11 to day $17(n=6)$. To explore the homeostatic turnover of pancreatic cells, two doses of $50 \mu \mathrm{g} / \mathrm{kg} \mathrm{BrdU}$ (at $2 \mathrm{~h}$ and $16 \mathrm{~h}$ ) were administered at day 18 (three mice for each group). At day 18, mice were sacrificed and processed biochemical and molecular analysis.

$(p<0.001$; Figure 5(a)). Body weight decreased in STZtreated mice from day $5^{\text {th }}$ of STZ treatment; the decrease was significant at days 9 and 10 relative to their baseline (Figure 5(b)). Histopathological analysis showed loss of distinct lobular and cellular boundaries, vacuolar degeneration, fibrosis, and marked decrease in islets of Langerhans size as well as decrease number of cells in islets of Langerhans in the diabetic group compared to the nondiabetic group (Figure $5(\mathrm{c}, \mathrm{i})$ ). The number of damaged cells was increased in the diabetic group compared to the control (Figure 5(c, ii)). Pancreatic insulin content as estimated by ELISA showed significantly lower levels in diabetic animals, relative to nondiabetic animals (Figure 5(d)).

3.4. MSC-Exo Attenuated Hyperglycemia and Pancreatic Injury in STZ-Induced Diabetic Animals. After 10 days of STZ injection, diabetic animals were randomly assigned into three treatment groups: PBS, MSCs, and MSC-Exo (Figure 4). The treatment was given for 7 consecutive days (T1 to T7). A reduction in blood glucose levels was observed after MSCs or MSC-Exo treatment from day 1 of treatment (T1) onwards; the reduction was found to be significant from day 5 of treatment (T5) compared to their baseline (Figure 6(b)). In PBS-treated diabetic mice (STZ+PBS group), blood glucose further rose from day 1 of treatment (T1) and remained significantly higher compared to MSC/ MSC-Exo-treated animals till day 7 (T7) (Figure 6(b)). Body weights did not change significantly during the treatment period (from T1 to T7, Figure 6(c)). Histological analysis showed recovery of the cellular architecture of islets of Langerhans, with the reduction in vacuoles and fibrosis in the MSC-Exo and MSC-treated group as indicated by the increased number of cells (Figure $6(\mathrm{a}, \mathrm{ii})$ ) and cellular density in islets of Langerhans compared to the PBS-treated group (Figure 6(a, i)). Although the boundaries of islets of Langerhans were not well defined, they were surrounded by a fine capsule with increased cellular density in diabetic mice treated with MSCs and MSC-Exo (Figure 6(a)).

3.5. MSC-Exo-Induced Proliferation of Islet Cells and Increased Pancreatic Insulin Production in Diabetic Animals. Proliferation induction in the pancreatic islets was observed in MSC and MSC-Exo-treated animals compared to the PBS-treated group (Figure $7(\mathrm{a})$ ), as indicated by increased BrdU-positive cells in islets of Langerhans $(p<0.001$ vs. PBS group; Figure $7(b))$. Moreover, the pancreatic insulin content (ELISA assay) estimated at the end of treatment (at T7) was significantly higher in the MSC and MSC-Exo-treated groups relative to the PBS group $(p=0.057$ vs. PBS group; Figure 7(c)).

3.6. Regeneration Pathways Were Differentially Regulated in the Pancreas of MSCs and MSC-Exo-Treated Animals. NGS analysis revealed out 43,000 genes, 667 differentially up 


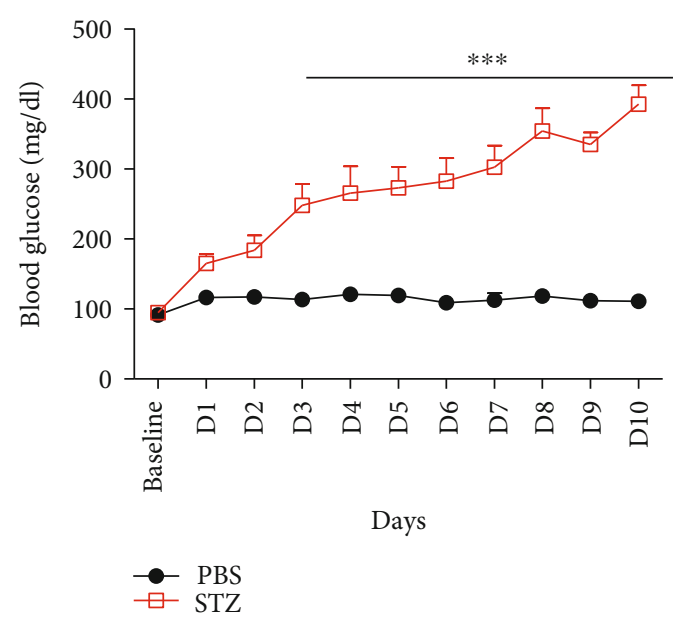

(a)

(i)

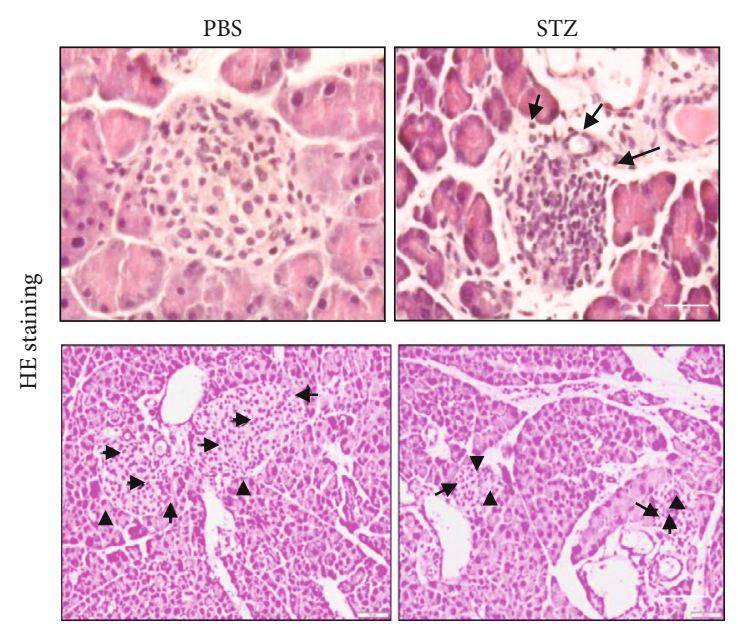

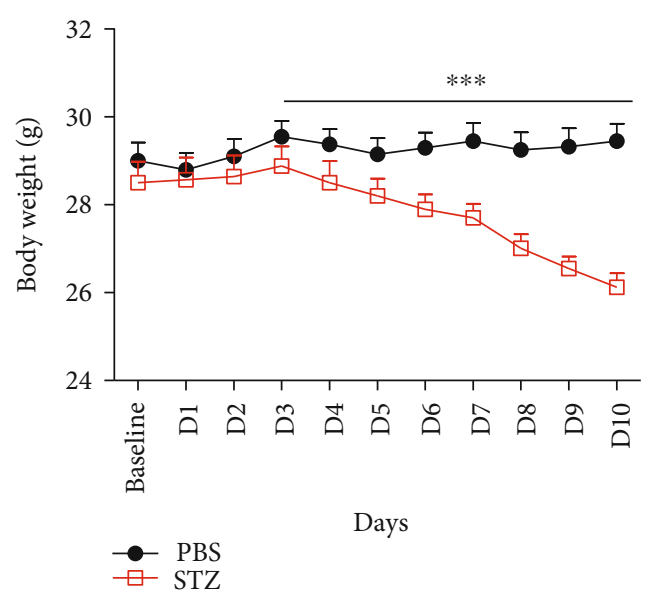

(b)

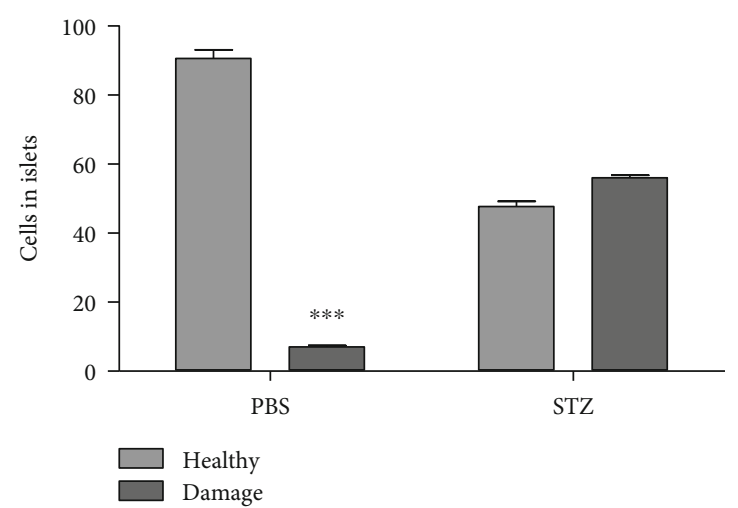

(c)

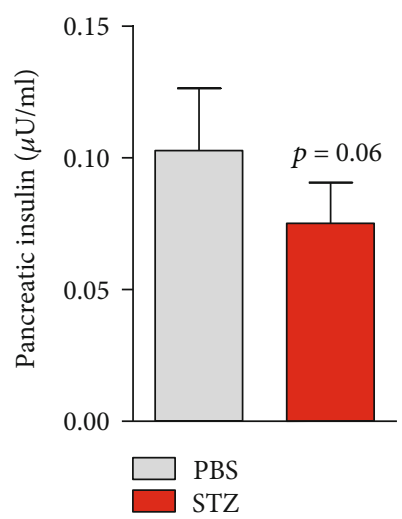

(d)

FIGURE 5: STZ treatment-induced diabetes in mice. (a) Line graph showing blood glucose levels in diabetic mice compared to the control animals over the experiment period ( $n=6$ /group). (b) Line graph showing body weight in the diabetic group compared to the control group ( $n=6 /$ group). (c) (i) Representative images of histological analysis of H\&E (scale bar $=20 \mu \mathrm{M}$ ) in the diabetic and control groups showing the altered pancreatic morphology. The lower panel shows images of H\&E staining with multiple islets (low magnification, scale bar $=100 \mu \mathrm{M}$ ). (c) (ii) Histogram representing the number of healthy and damaged cells in islets counted on three different sections (five random fields) in the diabetic group and control group. (d) Histogram representing insulin levels in pancreatic tissue of the diabetic and control groups. Data are shown as the mean $\pm S D$ ( $n=6$ /group). The significance of differences between mean values was estimated by nonparametric Student's $t$-test (unpaired, two-tailed). ${ }^{*} p<0.05$ and ${ }^{* * *} p<0.001$ compared to control animals. 
(i)

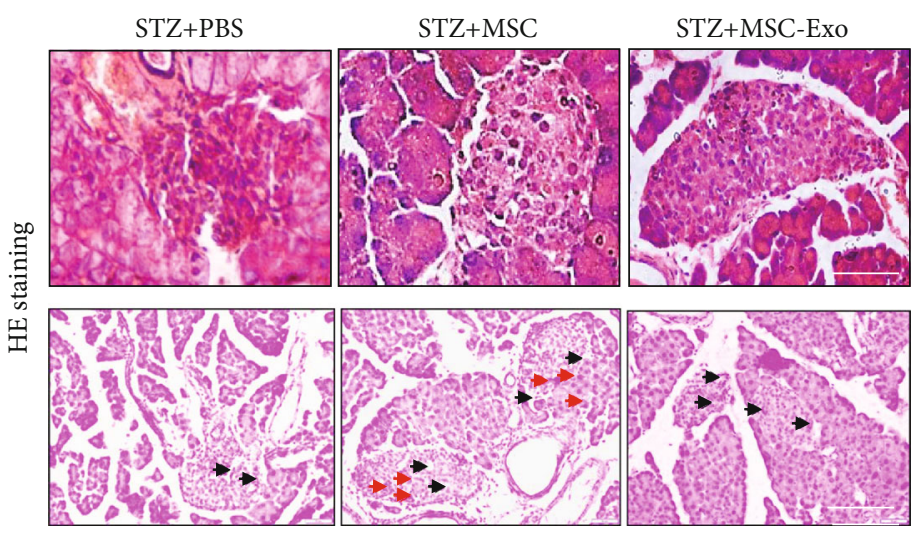

(ii)

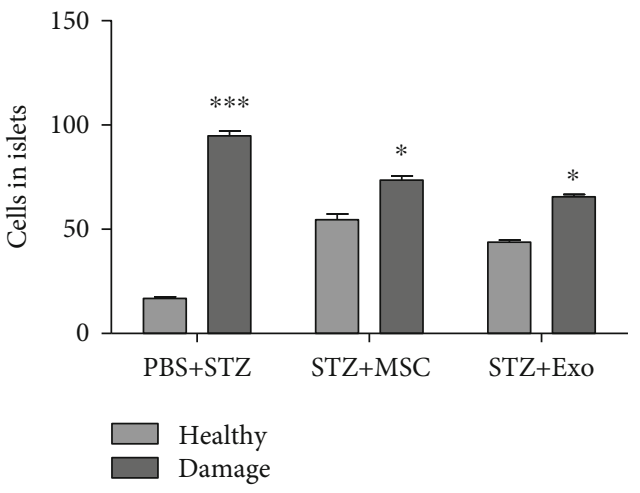

(a)

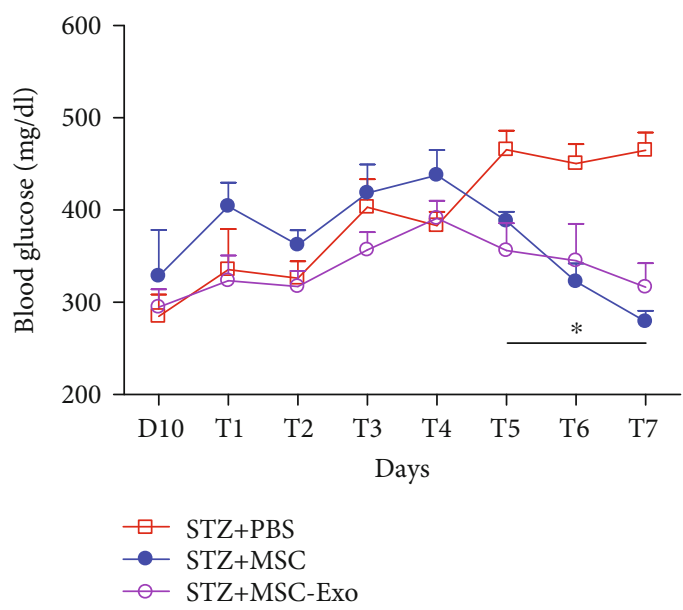

(b)

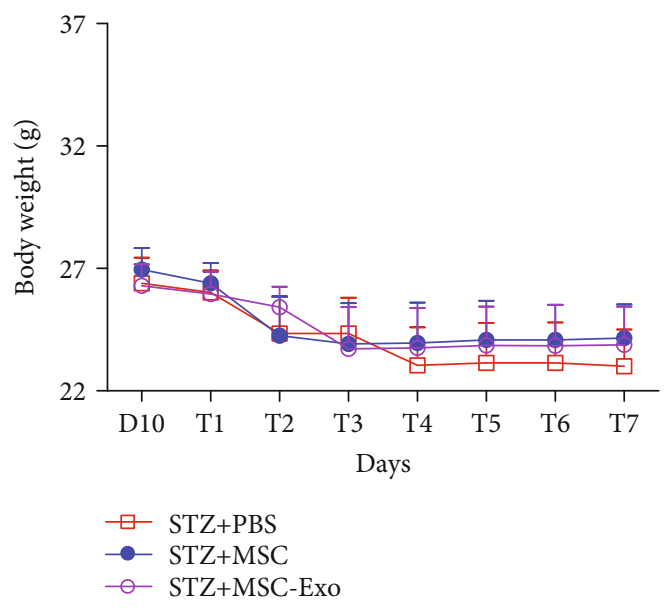

(c)

Figure 6: Reduced glucose levels in the MSC and MSC-Exo-treated groups. (a) (i) Representative images of H\&E staining (scale bar $=50 \mu \mathrm{M}$ ) of pancreatic tissue of the STZ+MSC and STZ+MSC-Exo groups compared to the STZ+PBS group. The lower panel shows images of H\&E staining with multiple islets (low magnification, scale bar $=100 \mu \mathrm{M}$ ). (ii) Histogram representing number of healthy and damaged cells in islets counted on three different sections (five random fields) in the STZ+PBS, STZ+MSC, and STZ+MSC-Exo groups. (b) Line graph showing blood glucose level in diabetic animals treated with the MSC and MSC-Exo groups compared to the PBS-treated group. (c) Line graph showing body weight in diabetic animals treated with the MSC and MSC-Exo groups compared to the PBS-treated group. Data are shown as the mean $\pm \mathrm{SD}$ ( $n=6$ /group). The significance of differences between mean values was estimated by nonparametric Student's $t$-test (unpaired, two-tailed) ${ }^{*} p<0.05$ and ${ }^{* * *} p<0.001$ compared to the PBS-treated group. 

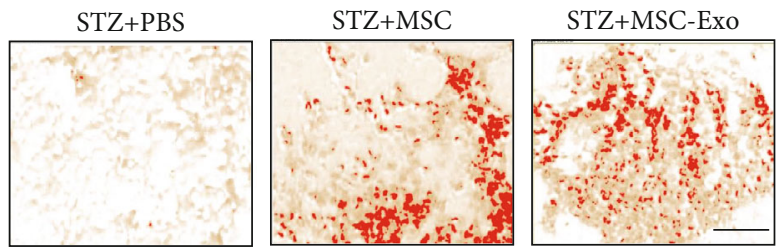

(a)

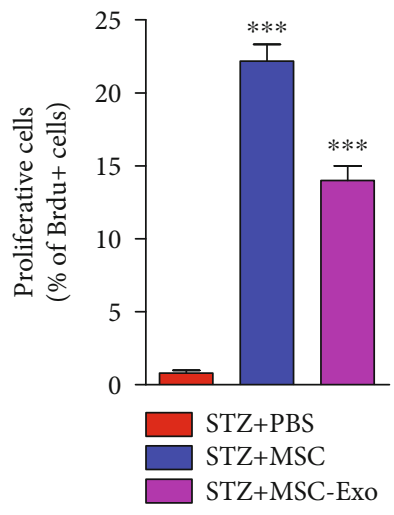

(b)

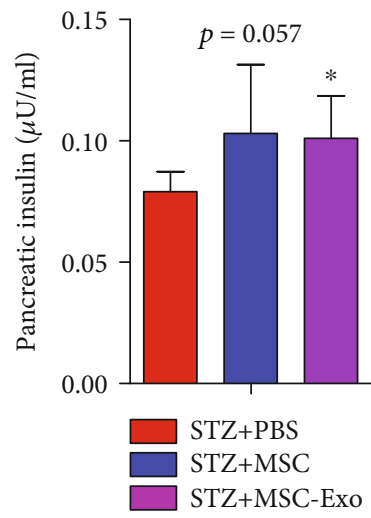

(c)

FIGURE 7: Intravenous post-MSC and Exo treatments increase cell proliferation and insulin level in the MSC and MSC-Exo-treated group. (a) Representative histological images of pancreatic tissue stained with BrdU showing increased BrdU-positive cells in diabetic animals treated with MSC or MSC-Exo in comparison with PBS-treated animals. Scale bar $=100 \mu \mathrm{M}$. (b) Histogram representing the percentage of BrdU-positive cells in MSC and MSC-Exo-treated diabetic animals in comparison with the PBS-treated group. (c) Histogram representing increased insulin level in pancreatic tissue of diabetic animals treated with MSC and MSC-Exo in comparison with PBStreated animals. Data are shown as the mean \pm SD ( $n=6$ /group). The significance of differences between mean values was estimated by nonparametric Student's $t$-test (unpaired, two-tailed). ${ }^{*} p<0.05$ and ${ }^{* * *} p<0.001$ compared to the PBS-treated group.

and downregulated genes (cut off $> \pm 2.0$ fold change), in the pancreatic tissue of diabetic animals treated with MSCs and MSC-Exo relative to the PBS group. The expressions of these differentially regulated genes were summarised in a heat map (Figure 8(a)), and pathway analysis of the differentially regulated genes showed involvement of insulin signaling and tissue regeneration pathways. Figure 8(b) shows genes, identified in our analysis, that have been associated with these pathways. A few of the identified genes were further validated using qPCR analysis, including Reg3 (regenerating islet-derived protein III), Reg2 (regenerating islet-derived protein II), and $A m y 2 b$ (amylase Alpha 2b) and TLR4 (Supplementary Table 1$)$.

\section{Discussion}

Restoring $\beta$ cell mass is an important management modality in diabetes [3-5]. Among several strategies for restoring $\beta$ cell mass, MSCs might serve as a promising agent due to their unique properties, including conditional differentiation, tropical support, and capabilities of spontaneous differentiation into connective tissue [24]. Moreover, they possess intrinsic immunosuppressive abilities [25]. MSCs derived from several sources such as bone marrow and dental pulp have been reported to attenuate diabetes and have pancreatic islet regeneration ability [26, 27]. Currently, the therapeutic potential of MSCs was extracted from studies based on the animal models; however, four clinical trials of MSCs in
T1DM are known till date, but due to smaller sample size, these results cannot be adequately evaluated and they are unable to demonstrate the significance as well [28]. Along with these, therapeutic use of MSCs may lead to cell-based therapy side effects and immune rejection. Recent studies [29-31] reported the promising role of exosomes in the treatment of T1DM. These studies suggested that MSCderived exosome could be a better alternative to MSCs due to their fewer side effects [29-31]. However, future studies and clinical trials are required to understand their mechanism of action. Moreover, the potential of MSC and MSCderived exosomes in attenuating endocrine deficiencies caused by nonautoimmune pancreatic $\beta$ cell loss needs to be tested. With this aim, we isolated and characterized MSCs from h-UCB (a noninvasive source of stem cells) based on their morphology, surface markers, and differentiation ability. After successful isolation of MSCs, we isolated the MSC-derived exosomes. Functional characterization of the MSC and MSC-derived exosomes was confirmed in vitro, where activated $\mathrm{T}$ cells were suppressed after treatment with MSC (79\%) or MSC-Exo (75\%). A similar report also showed the effect of MSC-Exo over MSCs in the rat model of T1DM [31]. Moreover, a therapeutic potential of stem cell-derived exosomal miRNA has been reported. Since the miRNA profiles of exosomes vary with type of stem cells, we compared the regulatory profile of these two treatment strategies (MSC and MSC-Exo) by comparing their miR profile. Using microarray analysis, we observed that hUCB- 


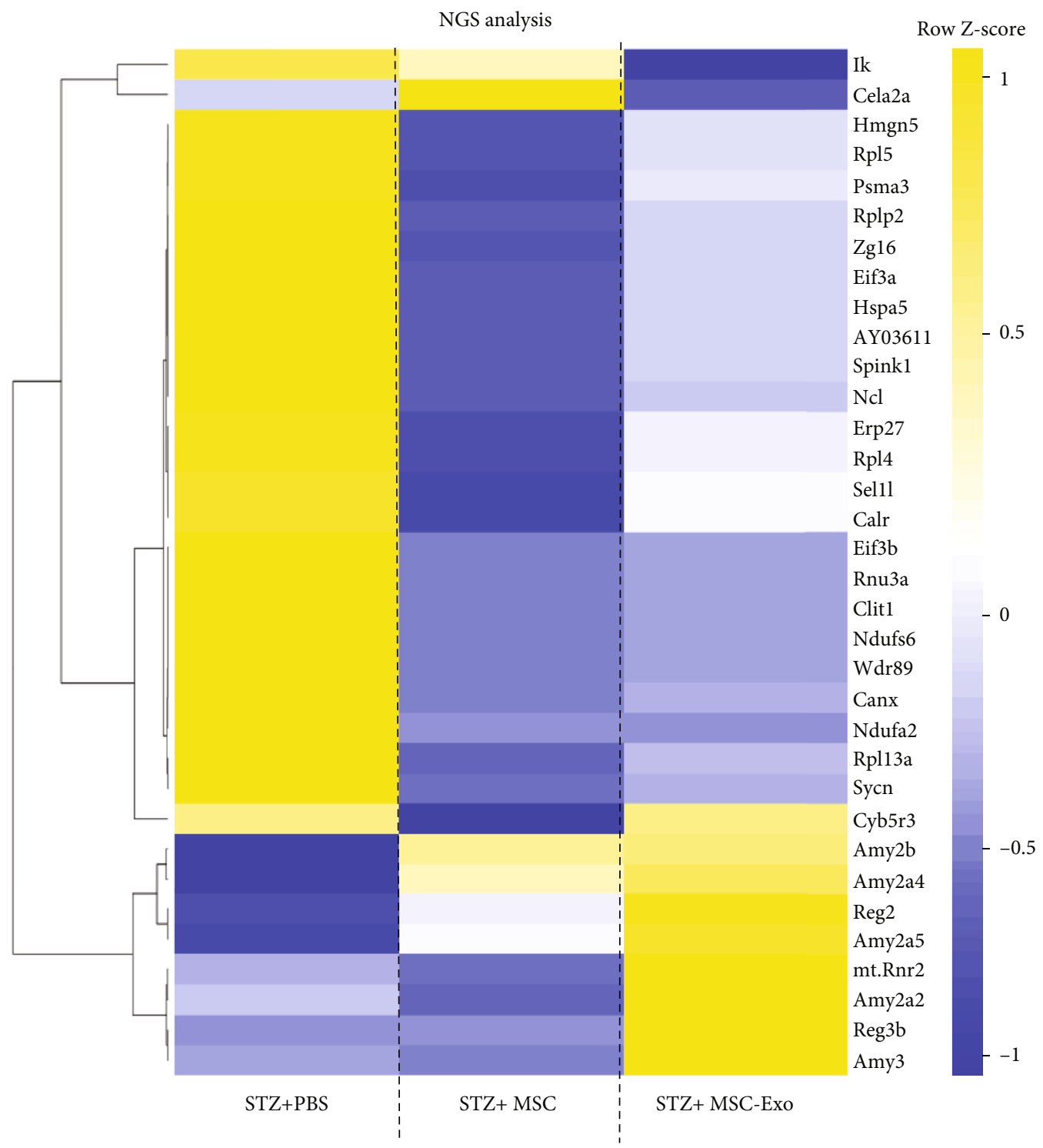

(a)

Figure 8: Continued. 


\begin{tabular}{|c|c|c|c|}
\hline \multicolumn{2}{|c|}{$\begin{array}{l}\text { Pathways related to } \\
\text { insulin signaling }\end{array}$} & \multicolumn{2}{|c|}{$\begin{array}{l}\text { Pathways related to } \\
\text { regeneration of cells }\end{array}$} \\
\hline $\begin{array}{l}\text { Pathways found in our } \\
\text { data }\end{array}$ & Gene & $\begin{array}{l}\text { Pathways found in } \\
\text { our data }\end{array}$ & Gene \\
\hline $\begin{array}{l}\text { MAPK signaling } \\
\text { pathway }\end{array}$ & 132 & Ribosome & 116 \\
\hline $\begin{array}{l}\text { PI3K-AKT signaling } \\
\text { pathway }\end{array}$ & 130 & $\begin{array}{l}\text { Oxidative } \\
\text { phosphorylation }\end{array}$ & 106 \\
\hline Ras signaling pathway & 126 & $\begin{array}{l}\text { Protein processing in } \\
\text { ER }\end{array}$ & 101 \\
\hline $\begin{array}{l}\text { Chemokine signaling } \\
\text { pathway }\end{array}$ & 104 & Cell cycle & 094 \\
\hline Insulin signaling & 067 & Apoptosis & 073 \\
\hline \multirow[t]{4}{*}{$\begin{array}{l}\text { Signaling pathways } \\
\text { regulating }\end{array}$} & 064 & RNA transport & 067 \\
\hline & 062 & $\begin{array}{l}\text { Carbon metabolism in } \\
\text { cancer }\end{array}$ & 048 \\
\hline & & Proteasome & 036 \\
\hline & & $\begin{array}{l}\text { Basal transcription } \\
\text { factors }\end{array}$ & 030 \\
\hline
\end{tabular}

(b)

FIgURE 8: Differential gene expression in pancreatic tissue of animals treated with MSCs and MSC-Exo. (a) Heat map representing differential gene expression in the pancreatic tissue of diabetic animals treated with MSCs and MSC-Exo in comparison with PBS-treated animals. $p<0.05$ was considered as the level of significance, and \pm 2 -fold change was the cut off value for heat map generation. (b) The table represents significantly differentially regulated pathways.

derived MSCs and their exosomes share similar miRNA profiles. We noted a huge expression of miRs that were known to be associated with cellular proliferation, cell cycle, inflammation, apoptosis, and metabolism pathways.

After successful isolation and characterization of MSC and MSC-Exo, we assessed their effect on pancreatic regeneration and the associated mechanism of action in vivo. STZ-induced diabetes and pancreatic injury were confirmed, respectively, by the induction of hyperglycemia and loss of pancreatic cellular architecture (diminution of islets of Langerhans' size and insulin content) compared to the control mice. Similar to our observation, Sabry et al. [31] reported high inflammation, vacuolar degeneration, congestion, and fibrosis along with the marked decrease in the size of islets of Langerhans. In our study, we have demonstrated regenerative benefits of MSCs derived from human UCB, in STZinduced diabetic mice. We found that both MSCs and MSC-Exo had similarly attenuated diabetes, as evidenced by decreased blood glucose levels after MSCs and MSCExo treatments. The treatments also promoted pancreatic regeneration compared to vehicle-treated diabetic mice. In support of our observations, study in rat pancreas demonstrated marked recovery of pancreatic architecture after treatment with MSC and MSC-Exo [31]. A similar beneficial effect of MSC-Exo, on STZ-induced diabetes, has also been reported in a recent study [32]. The author suggests that exosomes promote insulin sensitivity, increase glucose uptake and metabolism in peripheral tissues, and protect pancreatic islets from damage by inhibiting STZ-induced $\beta$ cell apoptosis. Recently, Mahdipour et al. have shown that exosomes derived from menstrual blood MSCs can regulate $\beta$ cell regeneration through PDX1-dependent mechanism in T1DM rat model [33].

Though the studies have confirmed the beneficial effect of MSC and MSC-Exo on pancreatic regeneration, but the mechanism remained illusive. Here, we performed transcriptome 
profiling in diabetic, MSC-, and MSC-Exo-treated pancreas. During transcriptome analysis of the pancreatic tissues, most of the transcripts $(\sim 80 \%)$ were found to be similarly regulated in MSCs and the MSC-Exo-treated group, relative to the PBS-treated group. However, a few of the transcripts were differentially regulated between MSCs and the MSC-Exotreated group. For example, Cyb5r3 (NADH cytochrome b5 reductase (b5R)), Reg2 (regenerating islet-derived protein II), Reg3b (regenerating islet-derived protein $3 \mathrm{~b}$ ), $m t R n r 2$ (mitochondrial encoded 16S rRNA2), Amy2a5 (amylase alpha 2A5), Amy2a2 (amylase alpha 2A2), and Amy2a3 (amylase alpha 2A3) genes were upregulated in the MSCExo group, but not in the MSC-treated group, as compared to the PBS-treated group. Moreover, Cela2a (chymotrypsinlike elastase 2A), Amy2a4 (amylase alpha 2A4), and Amy2b (amylase alpha $2 \mathrm{~b}$ ) genes were significantly upregulated in MSC-Exo relative to the MSC-treated group. The genes that regulated in the treated groups compared to the PBStreated group majorly belong to insulin signaling and tissue regeneration pathways. Within the insulin signaling pathway, the highest number of genes was associated with the MAPK signaling (132 genes) and the PI3K-AKT pathway (130 genes), including Ras, MAPKs, AKT, and IRS, while tissue regeneration pathway includes genes associated with the following processes: oxidative phosphorylation (106 genes), ER protein processing (101 genes), cell cycle (94 genes), ribosomal pathway (116 genes, predominantly Rpl 2-13, Rps 130, and Mrps 1-12), and proteasomal pathway (36 genes, Psmb, Psmd, and Psme (proteosomal subunit)).

Furthermore, by using qPCR analysis, we validated the upregulation of Reg3 (regenerating islet-derived protein III), Reg2 (regenerating islet-derived protein 2), and Amy $2 b$ (amylase alpha 2B) in the pancreatic tissue of the MSC and MSC-Exo-treated groups, in comparison to the PBS-treated group. Reg gene encodes an endogenous lectin which may be involved in the regeneration and growth of $\beta$ cells [34]. Reg 3 could also stimulate $\beta$ cell regeneration by reducing inflammatory conditions by the Jak-Stat 3 signaling pathway [34]. Thus, upregulation of Reg3 found in the pancreas of MSC-Exo-treated diabetic animals may have a role in the $\beta$ cell regeneration leading to the recovery of the insulin level [35-37]. Furthermore, an indispensable role of Reg protein in the pathophysiology of various human inflammatory diseases, especially $\beta$ cell regeneration in pancreatic inflammation damage models, has been demonstrated [37]. Reg is regulated by multiple genes, including Glp1 [38], PDX1 [33], Extl3 [39, 40], and PARP [41] and in turn affects multiple signaling pathways in a stimulus-dependent manner. Reg3a has been shown to regulate keratinocyte proliferation and differentiation after skin injury via Reg-Extl3-PI3KCyclin D1 pathway [42], while Reg3g downregulates the Stat3-Socs signaling which may result in enhanced apoptosis in acute pancreatitis [43]. The pathways analysis in our study revealed that most of the downstream targets of Reg2 and Reg3 were altered in the pancreas of MSC-Exo-treated animals. Moreover, several miRNAs regulating these signaling pathways were found in the UCB-MSC-derived exosomes, including miRNA-regulating Extl3 (miR-let-7a$5 p$, miR-24-3p, and miR-19-b-1-5p) and Cela2a (miR-450- b-5p) expression. Thus, miRNAs in the MSC-derived exosomes may facilitate regeneration by regulating the Extl3Reg-cyclinD1 pathway in the pancreas.

\section{Conclusion}

In conclusion, we have shown that exosomes derived from human UCB-MSCs significantly reduce blood glucose level, increase insulin production, limit pancreatic tissue damage, and improve disease outcomes in STZ-induced diabetic mice. Furthermore, exosome treatment-induced pancreatic tissue regeneration likely increased the Reg3 signaling pathways. Thus, UCB-MSC-derived exosomes may activate intrinsic regenerative abilities of pancreatic islets and attenuate insulin deficiency caused due to pancreatic cell destruction.

\section{Data Availability}

The datasets generated during and/or analysed during the current study are available from the corresponding author upon reasonable request.

\section{Ethical Approval}

For stem cells, the protocol has been approved by the Institutional Committee for Stem Cell Research (IC-SCR; Code: 2019-03-IMP-08). For animal models, the protocol has been approved by the Institutional Animal Ethics Committee (Ref. no: P-03/20/2017).

\section{Disclosure}

A part of the manuscript has been presented at the "10th Annual Meeting of the International Society for Extracellular Vesicles (ISEV)-2021, held in July 2021."

\section{Conflicts of Interest}

No potential conflicts of interest relevant to this article were reported.

\section{Authors' Contributions}

Rajni Sharma and Manju Kumari share equal authorship.

\section{Acknowledgments}

The authors wish to thank Dr. Krishna Kant, Dr. Kriti Joshi, and Ms. Medha Srivastava (Sanjay Gandhi Post Graduate Institute of Medical Sciences) and Dr. Drirh Khare (Hadassah-Hebrew University Medical Center, Ein Kerem, Jerusalem, Israel) for their technical assistance. This work was supported by Indo-Israel (grant no. DST/INT/ISR/P-23/ 2017) and Intramural (A-02-PGI/IMP78/2018) to ST.

\section{Supplementary Materials}

Supplementary Table 1: qPCR analysis for Reg2, Reg3, $A m y 2 b$, and TLR4. MSC-Exo treatment increases Reg2, Reg3, and Amy $2 b$ expressions. Along with this, MSC and 
MSC-Exo treatment attenuated STZ-induced increase in the level of TLR4 mRNA. Data represent as the mean $\pm \mathrm{SD} ;{ }^{*} p$ $<0.05$ versus the STZ+PBS group, by a two-tailed unpaired $t$-test. (Supplementary materials)

\section{References}

[1] M. Copenhaver and R. P. Hoffman, "Type 1 diabetes: where are we in 2017?," Transl Pediatr, vol. 6, no. 4, pp. 359-364, 2017.

[2] L. A. DiMeglio, C. Evans-Molina, and R. A. Oram, "Type 1 diabetes," Lancet, vol. 391, no. 10138, pp. 2449-2462, 2018.

[3] Q. Zhou and D. A. Melton, "Pancreas regeneration," Nature, vol. 557, no. 7705, pp. 351-358, 2018.

[4] K. A. Page and T. Reisman, "Interventions to preserve beta-cell function in the management and prevention of type 2 diabetes," Current Diabetes Reports, vol. 13, no. 2, pp. 252-260, 2013.

[5] V. Pathak, N. M. Pathak, C. L. O’Neill, J. Guduric-Fuchs, and R. J. Medina, "Therapies for type 1 diabetes: current scenario and future perspectives," Clinical Medicine Insights: Endocrinology and Diabetes, vol. 12, p. 1179551419844521, 2019.

[6] R. Abdi, P. Fiorina, C. N. Adra, M. Atkinson, and M. H. Sayegh, "Immunomodulation by mesenchymal stem cells: a potential therapeutic strategy for type 1 diabetes," Diabetes, vol. 57, no. 7, pp. 1759-1767, 2008.

[7] Q. Zhao, H. Ren, and Z. Han, "Mesenchymal stem cells: immunomodulatory capability and clinical potential in immune diseases," Journal of Cellular Immunotherapy, vol. 2, no. 1, pp. 3-20, 2016.

[8] K. Le Blanc and D. Mougiakakos, "Multipotent mesenchymal stromal cells and the innate immune system," Nature Reviews. Immunology, vol. 12, no. 5, pp. 383-396, 2012.

[9] L. Lin and L. Du, "The role of secreted factors in stem cellsmediated immune regulation," Cellular Immunology, vol. 326, pp. 24-32, 2018.

[10] A. Lernmark and H. E. Larsson, "Immune therapy in type 1 diabetes mellitus," Nature Reviews. Endocrinology, vol. 9, no. 2, pp. 92-103, 2013.

[11] M. M. Zanone, E. Favaro, I. Miceli et al., "Human mesenchymal stem cells modulate cellular immune response to islet antigen glutamic acid decarboxylase in type 1 diabetes," The Journal of Clinical Endocrinology and Metabolism, vol. 95, no. 8, pp. 3788-3797, 2010.

[12] E. Favaro, A. Carpanetto, C. Caorsi et al., "Human mesenchymal stem cells and derived extracellular vesicles induce regulatory dendritic cells in type 1 diabetic patients," Diabetologia, vol. 59, no. 2, pp. 325-333, 2016.

[13] P. O. Carlsson, E. Schwarcz, O. Korsgren, and K. le Blanc, "Preserved $\beta$-cell function in type 1 diabetes by mesenchymal stromal cells," Diabetes, vol. 64, no. 2, pp. 587-592, 2015.

[14] M. Wang, Y. Yang, D. Yang et al., "The immunomodulatory activity of human umbilical cord blood-derived mesenchymal stem cells in vitro," Immunology, vol. 126, no. 2, pp. 220-232, 2009.

[15] J. S. HEO, Y. CHOI, H. S. KIM, and H. O. KIM, “Comparison of molecular profiles of human mesenchymal stem cells derived from bone marrow, umbilical cord blood, placenta and adipose tissue," International Journal of Molecular Medicine, vol. 37, no. 1, pp. 115-125, 2016.
[16] E. H. Park, H. S. Lim, S. Lee et al., "Intravenous infusion of umbilical cord blood-derived mesenchymal stem cells in rheumatoid arthritis: a phase Ia clinical trial," Stem Cells Translational Medicine, vol. 7, no. 9, pp. 636-642, 2018.

[17] K. Bieback, S. Kern, H. Klüter, and H. Eichler, "Critical parameters for the isolation of mesenchymal stem cells from umbilical cord blood," Stem Cells, vol. 22, no. 4, pp. 625-634, 2004.

[18] B. Parekkadan and J. M. Milwid, "Mesenchymal stem cells as therapeutics," Annual Review of Biomedical Engineering, vol. 12, no. 1, pp. 87-117, 2010.

[19] S. Swioklo, A. Constantinescu, and C. J. Connon, "Alginateencapsulation for the improved hypothermic preservation of human adipose-derived stem cells," Stem Cells Translational Medicine, vol. 5, no. 3, pp. 339-349, 2016.

[20] W. Fu, X. Xie, Q. Li et al., "Isolation, characterization, and multipotent differentiation of mesenchymal stem cells derived from meniscal debris," Stem Cells International, vol. 2016, 2016.

[21] A. Kalani, A. Mohan, M. M. Godbole et al., "Wilm's tumor-1 protein levels in urinary exosomes from diabetic patients with or without proteinuria," PLoS One, vol. 8, no. 3, article e60177, 2013.

[22] K. K. Wu and Y. Huan, "Streptozotocin-induced diabetic models in mice and rats," Pharmacology, vol. 40, no. 1, pp. 5.47.1-5.47.14, 2008.

[23] A. A. Polak-Vogelzang, R. Hagenaars, and J. Nagel, "Evaluation of an indirect immunoperoxidase test for identification of acholeplasma and mycoplasma," Journal of General Microbiology, vol. 106, no. 2, pp. 241-249, 1978.

[24] A. Andrzejewska, B. Lukomska, and M. Janowski, "Concise review: mesenchymal stem cells: from roots to boost," Stem Cells, vol. 37, no. 7, pp. 855-864, 2019.

[25] J. Kobolak, A. Dinnyes, A. Memic, A. Khademhosseini, and A. Mobasheri, "Mesenchymal stem cells: identification, phenotypic characterization, biological properties and potential for regenerative medicine through biomaterial microengineering of their niche," Methods, vol. 99, pp. 62-68, 2016.

[26] D. Hess, L. Li, M. Martin et al., "Bone marrow-derived stem cells initiate pancreatic regeneration," Nature Biotechnology, vol. 21, no. 7, pp. 763-770, 2003.

[27] T. Izumoto-Akita, A. Yamamoto, E. Uenishi et al., "Secreted factors from dental pulp stem cells improve glucose intolerance in streptozotocin-induced diabetic mice by increasing pancreatic $\beta$-cell function," BMJ Open Diabetes Research \& Care, vol. 3, no. 1, article e000128, 2015.

[28] J. Cho, M. D'Antuono, M. Glicksman, J. Wang, and J. Jonklaas, "A review of clinical trials: mesenchymal stem cell transplant therapy in type 1 and type 2 diabetes mellitus," American Journal of Stem Cells, vol. 7, no. 4, pp. 82-93, 2018.

[29] B.-C. Lee, I. Kang, and K.-R. Yu, "Therapeutic features and updated clinical trials of mesenchymal stem cell (MSC)derived exosomes," Journal of Clinical Medicine, vol. 10, no. 4, p. 711, 2021.

[30] H. Pang, S. Luo, Y. Xiao et al., "Emerging roles of exosomes in T1DM," Frontiers in Immunology, vol. 11, p. 3099, 2020.

[31] D. Sabry, S. Marzouk, R. Zakaria, H. A. Ibrahim, and M. Samir, "The effect of exosomes derived from mesenchymal stem cells in the treatment of induced type 1 diabetes mellitus in rats," Biotechnology Letters, vol. 42, no. 8, pp. 1597-1610, 2020. 
[32] Y. Sun, H. Shi, S. Yin et al., "Human mesenchymal stem cell derived exosomes alleviate type 2 diabetes mellitus by reversing peripheral insulin resistance and relieving $\beta$-cell destruction," ACS Nano, vol. 12, no. 8, pp. 7613-7628, 2018.

[33] E. Mahdipour, Z. Salmasi, and N. Sabeti, "Potential of stem cell-derived exosomes to regenerate $\beta$ islets through Pdx-1 dependent mechanism in a rat model of type 1 diabetes," Journal of Cellular Physiology, vol. 234, no. 11, pp. 20310-20321, 2019.

[34] F. Xia, H. Cao, J. du, X. Liu, Y. Liu, and M. Xiang, "Reg3g overexpression promotes $\beta$ cell regeneration and induces immune tolerance in nonobese-diabetic mouse model," Journal of Leukocyte Biology, vol. 99, no. 6, pp. 1131-1140, 2016.

[35] K. Terazono, H. Yamamoto, S. Takasawa et al., "A novel gene activated in regenerating islets.," The Journal of Biological Chemistry, vol. 263, no. 5, pp. 2111-2114, 1988.

[36] T. Watanabe, Y. Yonemura, H. Yonekura et al., "Pancreatic beta-cell replication and amelioration of surgical diabetes by Reg protein," Proceedings of the National Academy of Sciences of the United States of America, vol. 91, no. 9, pp. 3589-3592, 1994.

[37] W. Cui, K. de Jesus, H. Zhao et al., “Overexpression of Reg3alpha increases cell growth and the levels of cyclin D1 and CDK4 in insulinoma cells," Growth Factors, vol. 27, no. 3, pp. 195202, 2009.

[38] J. A. Koehler, L. L. Baggio, X. Cao et al., "Glucagon-like peptide-1 receptor agonists increase pancreatic mass by induction of protein synthesis," Diabetes, vol. 64, no. 3, pp. 10461056, 2015.

[39] S. Kobayashi, T. Akiyama, K. Nata et al., "Identification of a receptor for Reg (regenerating gene) protein, a pancreatic $\beta$ cell regeneration factor"," The Journal of Biological Chemistry, vol. 275, no. 15, pp. 10723-10726, 2000.

[40] S. Yamada, "Specific functions of exostosin-like 3 (EXTL3) gene products," Cellular \& Molecular Biology Letters, vol. 25, no. 1, p. 39, 2020.

[41] T. Akiyama, S. Takasawa, K. Nata et al., "Activation of Reg gene, a gene for insulin-producing beta-cell regeneration: poly(ADP-ribose) polymerase binds Reg promoter and regulates the transcription by autopoly(ADP-ribosyl)ation," Proceedings of the National Academy of Sciences of the United States of America, vol. 98, no. 1, pp. 48-53, 2001.

[42] Y. Lai, D. Li, C. Li et al., "The antimicrobial protein REG3A regulates keratinocyte proliferation and differentiation after skin injury," Immunity, vol. 37, no. 1, pp. 74-84, 2012.

[43] M. Gironella, E. Folch-Puy, A. LeGoffic et al., "Experimental acute pancreatitis in PAP/HIP knock-out mice," Gut, vol. 56, no. 8, pp. 1091-1097, 2007. 